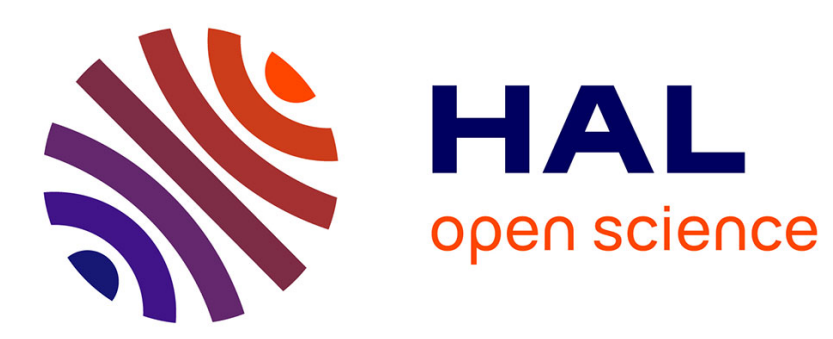

\title{
Les migrations des jeunes Sahéliens: affirmation de soi et émancipation
}

\author{
Mahamet Timera
}

\section{To cite this version:}

Mahamet Timera. Les migrations des jeunes Sahéliens : affirmation de soi et émancipation. Autrepart

- Revue de sciences sociales au Sud, 2001, 18, pp.37 - 49. 10.3917/autr.018.0037 . halshs-01497334

\section{HAL Id: halshs-01497334 \\ https://shs.hal.science/halshs-01497334}

Submitted on 28 Mar 2017

HAL is a multi-disciplinary open access archive for the deposit and dissemination of scientific research documents, whether they are published or not. The documents may come from teaching and research institutions in France or abroad, or from public or private research centers.
L'archive ouverte pluridisciplinaire HAL, est destinée au dépôt et à la diffusion de documents scientifiques de niveau recherche, publiés ou non, émanant des établissements d'enseignement et de recherche français ou étrangers, des laboratoires publics ou privés. 


\title{
Les migrations des jeunes Sahéliens: affirmation de soi et émancipation
}

\author{
Mahamet Timera *
}

Dans les migrations Sud-Nord, les jeunes hommes occupent une place prépondérante. Leur irruption dans l'espace public dépasse largement le cadre des pays du Sud et déborde dans les espaces des pays du Nord. Une des manifestations patentes de cette présence réside aussi dans leur mobilité qui traduit notamment une démarche conquérante de nouveaux mondes. Désir ardent et presque obsessionnel de mobilité auquel des cours tragiques mettent parfois fin de manière brutale, à l'instar du « demi-suicide » des deux jeunes garçons guinéens morts de froid dans le train d'atterrissage de l'avion qui devait les "arracher » à leur pays pour les conduire vers l'Europe. Comment qualifier un tel acte dont les auteurs ont cherché à expliquer le sens dans un pamphlet testamentaire en même temps que sorte de manifeste de la jeunesse du continent?

Aujourd'hui, la propension migratoire semble présente chez tous les jeunes du continent et concerne même des catégories encore dans l'adolescence. Dans certains pays du Sahel (Mali, Mauritanie, Sénégal), massivement la jeunesse des villes vient rejoindre celle de contrées rurales au passé migratoire plus ancien dans un projet commun: partir au Nord. Jamais projet n'a rencontré autant de suffrages au sein d'une jeunesse tant urbaine que rurale et nourri autant de rêves, de fantasmes et d'imaginaires. Bien que plus ou moins soutenus dans cette initiative par leurs familles et les parents déjà partis, la démarche d'émigrer n'en est pas moins discrète, parfois solitaire et secrète.

Partir pour « fuir la misère » et l'injustice, découvrir le monde, échapper à un univers étriqué, faire fortune, se former..., les mobiles qui guident les acteurs sont divers et multiples. Pourtant, il est difficile de dire que le migrant est l'affamé confronté au dilemme existentiel: partir ou mourir. Au demeurant, ceux qui sont soumis à une telle alternative ne partent généralement pas, faute de pouvoir le faire, et périssent sur place à moins qu'une opération humanitaire ne les sauve in extremis.

Quelle souffrance, sans doute aussi forte que la faim, anime donc ces jeunes qui prennent la voie de l'exil au prix parfois de leur vie avec comme credo: réussir ou y rester? Car on est loin souvent des situations de misère extrême. Prise en charge

* Chercheur associé au Centre d'études africaines (CEA-EHESS), Paris.

Autrepart (18), $2001: 37-49$ 
et solidarité familiales et communautaires épargnent à la plupart de ces jeunes une misère individuelle et une clochardisation.

Mais, si cette prise en charge autorise dans le pire le partage de la pénurie dans le cadre des solidarités familiales, empêchant ou retardant la misère comme sort individuel, n'exclut-elle pas par ailleurs pour ses bénéficiaires, notamment les cadets de sexe masculin, la reconnaissance, le respect et la considération auxquels ils aspirent légitimement?

Il semble que cette situation puisse se révéler éminemment pesante pour ses jeunes bénéficiaires; ce qui n'est pas le cas pour leurs aînés. En effet, si ces derniers en jouissent dans une sorte de droit à être secourus, entretenus, aidés, elle semble a contrario produire chez les jeunes hommes une souffrance plus forte ou plus intolérable que la faim, qui est celle, plus morale, de l'inexistence: le sentiment de «ne pas être », ou celui d'être réduit à ce que l'on ne désire surtout pas: l'insignifiance sociale.

Véritable phénomène d'anomie, l'impasse sociale, familiale et aussi biographique des jeunes pose l'exil comme issue possible. Le jeune candidat à l'émigration reste insatisfait de l'assistance familiale qui permet de survivre, voire de vivre décemment mais sans réelle considération familiale et sociale. Cette prise en charge familiale interdit de satisfaire non seulement des ambitions personnelles, mais surtout d'entrer dans le rôle social que la société attend de lui. Aussi le projet migratoire s'inscrit-il dans une volonté de rupture initiatique dans l'optique d'un futur retour glorieux permettant d'obtenir la reconnaissance sociale de son groupe et d'accéder à une véritable majorité sociale.

Rapportés à cette problématique de la réalisation ou de l'impossible réalisation individuelle et sociale, les projets migratoires des jeunes prennent tout leur sens et on comprend à bien des égards qu'ils se présentent comme un engagement extrême, un défi sans mesure apparente avec leurs conditions de vie matérielles réelles.

Ainsi, paradoxalement, si les solidarités familiales et communautaires conjurent des situations de misère sociale et matérielle aiguës, elles rendent possibles d'autres souffrances tout aussi indicibles par la violence qu'elles font subir à leurs jeunes bénéficiaires. Loin d'une vision simplement idyllique, la solidarité s'avère avoir un prix et constituer un lourd tribut à payer. Son envers est bien souvent le mépris ressenti et une minorisation sociale oppressante subie.

C'est au travers de cette approche biographique et familiale des jeunes garçons des villes au Sénégal et au Mali, des régions rurales de la vallée du fleuve Sénégal, que nous analyserons le sens des projets migratoires, leur fonction de consécration sociale et de modalité d'entrée dans l'espace public.

\section{Théories et représentations des migrations}

\section{Présentation des théories}

Dans l'approche des phénomènes migratoires du Sud vers le Nord se sont développés plusieurs modèles qui répondaient à des préoccupations scientifiques variées et correspondaient à des périodes différentes. Ainsi les premières approches ont cherché à analyser le phénomène migratoire dans le cadre de la pro- 
blématique des mouvements de main-d'œuvre, des processus de salarisation et de prolétarisation de populations paysannes et rurales du Sud, de leur intégration dans le capitalisme mondial [Amselle, 1976]. Les cadres conceptuels les plus élaborés et les plus fréquemment sollicités seront les théories de la division internationale du travail et de la dépendance, la théorie du mode de production domestique.

Pour les tenants de la théorie de la dépendance - Samir Amin, A. Gunder Franck -, la division inégale du travail avec la domination des «centres » du Nord sur les "périphéries » du Sud inscrit la migration des hommes notamment dans un schéma d'exploitation des premiers sur les seconds avec une relégation de la fonction d'entretien et d'élevage du travailleur migrant à l'économie domestique des pays d'origine [Meillassoux, 1975, rééd. 1991]. Ainsi, c'est une économie substantielle qui est réalisée par les pays d'immigration dans leur politique d'accueil des travailleurs étrangers. Indéniablement, ces théories citées ont permis de cerner la problématique du travailleur émigré et la dynamique de l'émigration dans ces sociétés.

Dans un autre registre, les théories du push and pull, d'offre et demande, et des avantages et coûts comparatifs, ont tenté de fournir une explication du phénomène migratoire. Si, à l'échelle macroéconomique, il y a une apparente évidence à établir une opposition entre la richesse du Nord et la pauvreté du Sud, à imaginer un marché du travail caractérisé d'une part par un excédent de main-d'œuvre au Sud et une pénurie au Nord, il faut relever qu'à la vérité, les choses ne se présentent guère ainsi et il n'existe pas de correspondance harmonieuse entre une offre du Nord et une demande du Sud. Les interférences entre le politique (contrôle des flux migratoires, fermeture totale ou partielle des frontières), les politiques salariales, une certaine liberté du marché de l'emploi, et les initiatives et projets des acteurs migrants construisent une résultante sociale plus ou moins fluctuante.

Du côté des représentations populaires tant des migrants du Sud que de larges secteurs de l'opinion publique, l'explication de la mobilité de certains groupes se fonde sur la misère des zones de départ. Un tel discours est du reste largement repris par certains acteurs politiques. Pourtant, les représentations qui associent les migrations à la misère et à la famine, pour tenaces qu'elles soient, ne correspondent pas à la réalité. Ce sont rarement les populations des zones les plus pauvres qui émigrent le plus. Émigrer est jusqu'à un certain point un luxe qui n'est pas à la portée de tous. Certes, une certaine hantise du Nord, plus ou moins prisonnier d'un imaginaire collectif, pousse d'aucuns à voir arriver aux portes de l'Europe des hordes d'affamés du Sud. Mais les figures produites par les jeunes migrants tranchent avec ces caricatures.

Ainsi, dans une remise en cause de ce paradigme, l'histoire des migrations au départ de la région du haut-Sénégal révèle que, dès le XIX ${ }^{\mathrm{e}}$ siècle, on enregistrait des installations en Afrique centrale sans que ces mouvements ne correspondent à une situation économique catastrophique de la région de départ [Manchuelle, 1986]. Dans une période plus récente, Catherine Quiminal, retraçant l'histoire migratoire de trois jeunes, écrit:

« Hamédy, Sow et Kamara ont décidé d'aller travailler en France. Et pourtant, en 1969, la récolte n’a pas été mauvaise dans la région de Kayes, tout le monde mange au village. [...] 
Ce n'est pas la famine ou la misère qui ont poussé Hamédy, Sow et Kamara à quitter leur famille, à quitter le village. Du moins, ce n'est pas ainsi qu'ils voient les choses » [Quiminal, 1991: 10].

C'est dire que la corrélation rapide entre pauvreté et misère d'une part, et émigration de l'autre, doit être révisée de façon critique car elle est insuffisante et parfois fausse pour rendre compte des causes de l'émigration.

À mi-chemin entre les représentations populaires et le discours savant, se dégage la vision culturaliste qui définit la migration comme un " trait culturel », une pratique intégrée dans un système de valeurs et un mode de vie spécifique (les peuples de voyageurs). Cette réputation de "grands voyageurs " accordée à ces populations a aussi alimenté chez certains auteurs une vision de cette «tradition » du voyage fonctionnant comme un "rite initiatique ». Si une telle vision peut paraître intéressante comme perspective, elle reste, néanmoins un peu réductrice pour expliquer le phénomène migratoire. Nous pensons qu'il a été fait un sort trop rapide à cette approche de la migration comme rite initiatique et fait de culture. On peut à juste titre postuler qu'à la longue, et sans évacuer les déterminants économiques ou sociaux à l'origine du phénomène, que la migration puisse devenir aussi un fait de culture, tant elle s'intègre dans les pratiques d'une société et dans l'histoire individuelle.

Dire que la migration est une donnée culturelle n'en exclut pas l'historicité et ne lui ôte pas son caractère de fait social historiquement produit. Nous assistons ainsi, pour ces sociétés de la vallée du fleuve Sénégal plus concernées par ce type d'approche, à la création d'un " rite moderne » à travers la migration des hommes vers l'Europe, comme cela fut aussi le cas antérieurement vers le bassin arachidier. Il en est de même pour d'autres régions du pays Wolof où apparaît une émigration structurelle vers l'Italie par exemple. Celle-ci tend à devenir un phénomène majeur dans la socialisation et l'accès à un statut reconnu pour les jeunes. Certes, il s'agit plus d'un « rite de passage » inégalitaire qui construit une différenciation à la fois familiale et sociale.

À l'autre versant du procès migratoire et de la dialectique émigration/immigration [Sayad, 1991; 1999] correspond la problématique de l'intégration et/ou exclusion dans la société française. Autour de cette préoccupation et avec l'observation et l'analyse du processus d'insertion des migrants, se sont construits plusieurs modèles d'interprétation dont les plus représentatifs s'inspirent de l'école de Chicago [Grafmeyer, Joseph, 1984, $2^{\mathrm{e}}$ éd.], de l'approche interculturelle, des relations interethniques et « raciales » [de Rudder, 1992; Wieviorka, 1992].

\section{Pour un retour à la dimension individuelle de la migration}

Ce qui nous a paru relativement absent dans ces théories et ces travaux, c'est la dimension individuelle de la migration, sa fonction d'émancipation, d'autonomisation et d'individualisation. L'analyse du phénomène migratoire dans la région de la vallée du fleuve Sénégal a surtout mis l'accent sur les modalités de la prise en charge collective de la famille élargie d'abord, du village par la suite. La migration comme démarche individuelle d'autonomisation et d'émancipation des acteurs a très peu été abordée, voire perçue ou acceptée, par les chercheurs ayant travaillé 
sur cette question. Ainsi nous avons, dans nos travaux antérieurs [Timera, 1996], défini le migrant davantage comme un "envoyé », le messager de toute une communauté, mû par les intérêts supérieurs de ses «mandants » restés au village. Ce faisant, certains se sont demandé à juste titre si les migrants n'étaient pas exploités par leurs compatriotes restés au pays, eu égard aux souffrances qui étaient les leurs en France et compte tenu de toutes leurs réalisations et prestations pour les familles et le village natal. Une méthode d'analyse centrée sur le groupe et les pratiques de groupe et très peu sur l'acteur individuel, une certaine dépendance par rapport à des problématiques militantes et « développementalistes » sur un sujet politiquement et idéologiquement chargé et, enfin, un relatif emprisonnement sinon une forte empathie et sympathie avec le discours des migrants expliquent jusqu'à un certain point ces biais dans notre approche.

Les migrants de la vallée procèdent, en même temps qu'ils prennent en charge la grande famille et le village, à des réalisations plus individuelles: construction, acquisition de biens immobiliers, épargne individuelle, entreprise personnelle, prise en charge particulière de leur ménage ou des parents directs au sein du patrilignage: femmes et enfants, ascendants, frères et sœurs utérins... Et pourtant, la recherche reste timide et quasiment muette sur ces faits. Tout se passe comme si ce champ des pratiques était insignifiant ou n'entrait pas dans l'analyse du fait migratoire ou alors de manière seulement incidente. Quelques travaux comme ceux de S.M. Tall [1994] ont, du côté des pays d'origine, étudié les investissements des migrants - en général des acquisitions individuelles - dans le secteur de l'immobilier. Cet accès à la propriété représente un des objectifs majeurs des migrants, souvent réalisés en milieu urbain et consécutifs à la réhabilitation de la maison paternelle (construction en dur). De fait, il constitue une voie vers l'individuation, prépare une décohabitation par rapport à la famille élargie et favorise la privatisation des biens.

Avec l'entrée dans le salariat, premier levier et élément essentiel dans ce processus d'affirmation individuelle, les revenus acquis par ce biais seront en partie cristallisés dans un patrimoine personnel et non intégralement investis dans l'économie collective.

À ne voir que les entreprises collectives des migrants, parce qu'elles ont été construites et posées comme les objets de recherche, nous nous sommes interdit de voir autre chose et avons appauvri l'analyse du phénomène.

Plus que d'être seulement le fait d'un biais méthodologique et/ou idéologique du chercheur, cette absence d'attention portée à la dimension plus personnelle du projet migratoire est également le fait de la société et des acteurs eux-mêmes. Non pas qu'ils fassent preuve de duplicité en mettant en avant la seule dimension collective de la migration ou qu'ils remettent en cause toute démarche de promotion individuelle, de différenciation et d'individuation. En vérité, dans la mesure où la perspective intègre la solidarité avec la collectivité et non la rupture, elle est légitimée. Mais, l'affirmation simultanée de ces deux projets contribue à brouiller un tant soit peu les données. Ainsi, même si la migration participe réellement de la promotion de l'individu, de la différenciation et, partant, de l'introduction de nouvelles formes d'inégalités (et de violence) dans la société, l'affirmer comme telle pour les acteurs apparaît comme quelque peu contradictoire avec les valeurs dominantes. 
D'autant plus contradictoire que la migration fait souvent l'objet d'une prise en charge familiale, se réalise grâce à cette solidarité et appelle implicitement une attitude reconnaissante. La dimension compétitive, individualisante, de la migration est à la fois un refoulé et un univers quasi secret. Même au sein des patrilignages, des zones d'ombre soustraient du regard de la collectivité des projets, des entreprises et des situations individuelles. On l'aura compris, ce qui se joue ici porte au fond sur les dynamiques de différenciation qui traversent la famille. Comment concilier sa fonction solidaire et communautaire qui s'exprime concrètement par l'unité de résidence et de consommation en général en milieu urbain, à laquelle s'ajoute l'unité de production en milieu rural avec les aspirations et stratégies individualistes qui la traversent?

Il apparaît que ces sociétés africaines n'ont nullement eu pour projet de nier l'individu comme réalité en leur sein. Mais, ce qui importe, c'est davantage la finalité de cette individuation et/ou différenciation: donner et rendre à la communauté ce que l'on a soi-même reçu, « rembourser » et prêter dans une logique sans fin [Marie, 1999].

Mais donner, c'est avoir, s'approprier de façon privative, accumuler, et l'affirmation individuelle se réalise à la fois dans l'acte d'avoir et de donner. La souffrance des jeunes est autant de ne pas avoir que de ne pas pouvoir rendre aux aînés. Cette logique du don et du contre-don, du remboursement de la "dette sociale ", structure les relations interpersonnelles dans l'espace familial, mais aussi dans l'espace public communautaire ou local, voire national. Les jeunes adultes sont, en tant que cadets, fortement tributaires de cette logique. Et, alors qu'arrive le moment de la restitution, leur tour de donner, ils vivent une crise de mobilité qui les confine dans une impossibilité d'être socialement. C'est particulièrement le cas des jeunes citadins qui, à la différence de leurs homologues ruraux ne peuvent, par une mobilisation dans les travaux agricoles, rendre ou commencer à rendre ce qu'ils ont reçu. Pour les jeunes citadins, la seule vraie reconnaissance passe par l'accès à l'emploi rémunérateur. C'est la condition de leur entrée dans l'espace public comme individus majeurs ayant un statut social.

\section{L'émancipation et la réalisation par le départ}

À travers la migration, les jeunes ruraux cherchent un moyen pour accéder à des biens et revenus propres. Ils tentent de contourner le système communautaire, recherchent l'individuation et refusent la solidarité familiale unilatérale et perpétuelle, fût-elle à leur bénéfice, car elle les infantilise et les minorise. L'étude du système de production, de consommation et d'échanges dans les sociétés de la vallée du fleuve Sénégal et la place conférée aux jeunes informent sur la nature réelle de l'espace économique qui leur est dévolu. La production et la consommation se réalisent dans la structure sociale communautaire que constitue la concession familiale (patrilignage). La propriété des moyens de production essentiels (la terre) est collective tout autant que leur mise en valeur. Généralement, la terre est un bien indivisible et inaliénable dont la gestion, pour le bénéfice du patrilignage bien sûr, est assurée par le doyen. À l'intérieur de la cellule de production ainsi définie, une bonne part de la rente migratoire est gérée par le doyen dont la fonc- 
tion est d'assurer le fonctionnement du patrilignage: diriger la production, assurer les repas quotidiens, régler l'impôt..., bref tout ce qui relève de la collectivité. Les besoins plus individuels sont très souvent laissés à la charge des intéressés.

Ainsi, si les jeunes sont pris en charge pour la nourriture et l'hébergement, ils se trouvent privés de la gestion d'un espace économique individuel et privé. Alors que l'économie marchande pénètre de plus en plus leur espace à travers des objets de consommation devenus à la longue indispensables, tous les autres besoins qui ne relèvent pas de la reproduction immédiate sont pour ainsi dire éludés.

Pourtant, ces sociétés locales avaient perçu cette exigence d'un espace économique individuel et allouaient aux dépendants majeurs et aux jeunes adultes une parcelle de terre sur laquelle ils pouvaient travailler pendant une partie de la journée, une fois leur prestation sur le champ collectif familial terminée. Quoique disposant d'un moindre temps de travail et de main-d'œuvre, l'existence de ces parcelles témoigne de la prise en compte d'un besoin social. Cette articulation de l'espace économique collectif avec l'existence d'espaces privés individualisés répondait à cette exigence fondamentale ${ }^{1}$. Or, avec la détérioration des conditions agricoles et la baisse des récoltes, avec également le resserrement de la maind'œuvre disponible suite à la multiplication des départs en émigration, le maintien des parcelles individuelles dont la rentabilité était somme toute faible devenait de plus en plus aléatoire.

Pour faire face au besoin vital de la reproduction domestique, toute la maind'œuvre sera concentrée pendant tout le temps de production sur le champ collectif. Situation qui renforcera le dénuement des jeunes et dépendants, la précarité de leur état, même si, grâce à la rente migratoire et à la prise en charge familiale, les jeunes sont loin des situations de misère. Néanmoins, la contrepartie de ces services est leur mise à contribution comme main-d'œuvre " gratuite " pour l'économie domestique collective. On comprend alors mieux les propos des jeunes sur leur «pauvreté ». En fait, ces propos doivent être entendus dans le sens de « n'avoir rien à soi » et non dans celui de la misère ou de la famine. L'exclusion d'un accès privatif aux biens et produits de l'économie familiale rend ainsi sans intérêt une augmentation des ressources et une modernisation des moyens de production de cette économie familiale. Pis, elle peut constituer un danger et une entrave à leur projet de départ en renforçant leur implication dans la production. C'est le constat que nous avons pu faire au cours de plusieurs enquêtes en pays Soninké dans la vallée du fleuve Sénégal et au Mali.

En effet, alors que les aînés et les chefs de concession militent clairement pour une modernisation de l'agriculture et des moyens de production face aux bailleurs de fonds et aux pouvoirs publics, en incitant d'ailleurs les jeunes à partager ce point de vue, ces derniers ont pour perspective la migration. Pourtant, leur souhait n'est pas de rompre avec l'unité familiale comme pourrait le suggérer cette mise à distance de ces propositions de modernisation et de développement. Au contraire,

1 Ailleurs et en d'autres temps, le système collectiviste de l'ex-Union soviétique avait permis l'existence de lopins de terre individuels à côté des terres collectives pour stimuler l'enthousiasme des producteurs socialistes. 
ils affirment vouloir partir pour rapporter quelque chose dans la maison paternelle, pour contribuer à son enrichissement.

«Un jeune homme doit partir chercher quelque chose pour le rapporter à la maison paternelle.»

«Chez les Soninké, la migration est une surprise qui peut toujours vous tomber dessus. »

Ces propos de jeunes rencontrés au Mali expriment avec force la permanence du fait migratoire dans cette société. Si les motivations des jeunes ne constituent certes pas une explication suffisante du phénomène migratoire, elles rendent compte de façon nette de la manière dont l'expérience est vécue, donnent un sens à leurs pratiques d'acteurs.

«Il n'y a rien ici », «c'est dur ici », « il n'y a pas de pluies », « l'agriculture ne rapporte pas », «on ne peut pas en vivre».

Aussi partent-ils " pour trouver quelque chose comme les autres déjà partis ", " pour résoudre leurs besoins », "pour aider les parents et les leurs, contribuer au développement du fabanka (la maison paternelle) », « pour se marier ».

Toutes ces expressions reviennent comme un leitmotiv dans la bouche des jeunes et traduisent le sentiment général que cette terre est ingrate et dure pour ses enfants.

Ils partent, mais ils ne rompent pas avec la famille ou rarement. Le projet n'est pas de couper tous les liens avec le village et les leurs, il est de partir pour essayer de "faire fortune » et seul l'attachement à la famille et au village peut donner sens à la réussite. Les différents acteurs dans la chaîne migratoire confirment ce lien du migrant avec le pays natal. Comme l'expriment ces propos d'un logeur à Bamako qui a vu passer chez lui des dizaines de migrants en transit pour l'étranger:

«Le Soninké est comme la perdrix, il a beau s'éloigner de son nid, il y retourne toujours. »

La richesse ne devient vraiment une valeur sociale que lorsqu'elle est investie dans la maison natale. Ailleurs, elle est sans intérêt social, donc sans intérêt tout court. On revient d'autant plus qu'on aspire à faire comme ceux qui, partis avant sont revenus au village, ont gagné la considération des leurs et acquis un statut plus élevé dans la communauté.

Partir, c'est souvent d'abord quitter le village pour un autre village, en général soninké, au Mali, au Sénégal ou en Mauritanie. Partir, c'est ensuite quitter le village pour la ville proche ou plus fréquemment la capitale, Bamako, Dakar. Partir enfin, c'est aussi quitter le village pour un autre pays d'Afrique ou pour l'Europe. Dans ces différents cas de figure, les migrations ont des caractéristiques et des fonctions spécifiques.

Le projet de migration est présent chez les jeunes ruraux et programmé à court, moyen ou long terme. On peut, en attendant sa réalisation, s'investir dans un projet individuel ou collectif au village, mais l'implication dans des activités rémunératrices est encore peu développée chez les jeunes. À titre d'exemple, sur une centaine de jeunes interrogés lors d'une enquête au Mali (région de Kayes) 
en 1995, seuls 17 avaient une activité individuelle rémunératrice dans les domaines suivants:

- petit commerce: 7 ,

- artisan tailleur: 2,

- réparateur radio: 1 ,

- vente de bois de chauffe: 2 ,

- gérant de moulin à mil: 1 ,

- maraîchage: 4.

Parmi ces activités, beaucoup sont intégrées dans l'économie domestique et les jeunes sont alors utilisés comme main-d'œuvre plus ou moins rémunérée et intéressée aux gains et sont rarement les premiers répondants de "l'affaire ». Ce statut de "semi-propriétaire »n'est certes pas de nature à assurer aux jeunes une réelle autonomie et se présente davantage comme un élargissement de l'économie domestique dont le premier gérant est le chef de la concession.

C'est pourquoi cette occupation ne supprime pas le désir et la volonté de partir. En dehors de l'économie domestique, quelques jeunes entreprennent une activité individuelle dans le village (maraîchage, commerce, artisanat) parallèlement à leur participation à l'économie familiale (champs et travaux familiaux) et arrivent à se constituer des revenus propres. Tous affirment néanmoins sans détours leur intention de partir eu égard aux faibles revenus que procurent leurs activités, à l'étroitesse du marché et à la carence des moyens et outils de travail. En somme, ces projets individuels organisent l'attente en vue d'un prochain départ.

Dans les projets migratoires, la France arrive largement en tête des destinations recherchées, mais on note une diversification vers les autres pays d'Europe de l'Ouest, les États-Unis et l'Afrique. Le projet migratoire est dans tous les cas situé hors du pays, et les seules fois où Bamako est citée comme destination correspondent à des sujets qui n'ont encore jamais quitté leur village et dont le désir immédiat est de partir à la capitale.

Le choix préférentiel pour la France se justifie par la présence de parents (pères, oncles, frères, cousins et amis) dans ce pays. Cette destination se présente presque comme naturelle tant la filière villageoise et familiale est ancienne et bien implantée. La France apparaît aussi comme le pays où, malgré les difficultés, on peut trouver plus rapidement qu'ailleurs du travail, gagner de l'argent et réaliser quelque chose. Ceux qui ont déjà « trouvé le chemin » en constituent la preuve vivante. Grâce à la migration en France, ils acquièrent un pouvoir économique et un statut social qu'une activité au village ou dans un autre pays d'Afrique peut difficilement offrir.

Le choix de la destination est rarement le fait du hasard ou de la subjectivité. Il répond à des considérations de contextes concrets: l'existence de filières familiales déjà constituées et la disponibilité de "points de chute ". Ainsi, on part souvent pour rejoindre quelqu'un de la famille déjà installé. On peut partir parce que fonctionnent des stratégies migratoires familiales qui font que le premier arrivé permet à ceux qui le suivent (fils, frères, cousins) de "passer ». La présence dans presque tous les cas d'un membre de la famille (père, oncle, frère, cousin) dans le lieu d'immigration choisi le confirme. 
Si les migrations vers d'autres régions proches ou vers les villes africaines peuvent se réaliser grâce à une accumulation individuelle, la migration de ces jeunes ruraux vers l'Europe se réalise grâce au soutien de leurs prédécesseurs. Ce n'est guère par une accumulation individuelle que se concrétise le projet. On part aidé, pris en charge. C'est un projet qui dépend financièrement des aînés déjà partis. D'où toute une négociation et bien des pressions des cadets pour convaincre les aînés de les aider dans la réalisation de leur projet.

Assistés, otages et main-d'œuvre plus ou moins gratuite de l'économie familiale, les hommes jeunes cherchent une autonomisation dans la migration. Mais la réalisation de cette perspective fait également appel à la structure familiale. La migration se présente comme un des moyens privilégiés de promotion au sein de l'espace familial et de l'espace public.

L'accès à cette promotion sociale n'est pas fonction que de l'âge et les jeunes voient bien que pour " grandir » et compter aux yeux du groupe, domestique et villageois, pour avoir un statut social valorisant public et privé (époux, aîné, fils prodige, etc.), il faut « acquérir » pour soi-même et en faire profiter les autres.

Ainsi, si la migration est une stratégie collective, familiale, voire villageoise, elle est également une entreprise éminemment individuelle dont une fonction majeure est l'individuation statutaire. Elle introduit la concurrence entre les individus, la différenciation à partir de l'accès inégal à des ressources qu'elle tempère par ailleurs, jusqu'à un certain point en assumant une charge solidaire importante. Aussi, si la migration dans le court terme renforce le système familial lignager, à plus longue échéance, elle le remet en cause.

Enfin, la migration offre le moyen de contourner les aspects infériorisants liés à cette structure familiale collective et collectiviste dans certains aspects, l'envers oppressant de la solidarité familiale.

Quoique la situation soit légèrement différente dans les villes, les projets migratoires des jeunes sont tout aussi affirmés. La mobilisation des citadins pour la migration est bien plus tardive et correspond à de réels problèmes de mobilité sociale et de socialisation dans l'espace familial et public, apparus notamment dans les années quatre-vingt. L'application des plans d'ajustement structurel du FMI, la crise de l'école avec le chômage massif des diplômés de l'enseignement supérieur, la remise en cause des " privilèges » des citadins (subvention des prix des denrées de première nécessité), le marché de l'emploi quasiment fermé posent avec acuité la problématique de la socialisation des jeunes et de leur émancipation sociale.

La prise en charge familiale bien que toujours présente se fait plus chiche eu égard aux faibles ressources. Certes l'hospitalité familiale fonctionne encore: le couvert et le gîte étant assurés tant bien que mal, la structure familiale garantit l'unité de résidence et la commensalité jusqu'à un certain point. Si les jeunes en milieu rural constituent, en contrepartie de leur prise en charge familiale une maind'œuvre au service du lignage, en ville, leur statut d'inactifs est d'autant plus manifeste qu'ils sont improductifs. Une telle situation souligne chez ces jeunes tout le poids des prises en charge dont ils font l'objet de la part de la communauté. Investissement communautaire qu'ils supportent comme une dette morale.

Leur seule possibilité de mise à contribution réside dans l'accès à l'emploi et à des revenus. Mais, pris en charges, éduqués, envoyés à l'école pour servir plus tard 
les leurs, ils en ressortent parfois diplômés mais sans emploi. Ainsi, la conjoncture actuelle rend problématique et hypothétique leur capacité à honorer cette dette et les réduit à l'état d'éternels assistés. Cette situation les confine dans une impossible réalisation sociale, une impossible entrée par la grande porte dans l'espace public. Cette impasse compromet tout un cycle social. Incapables de renvoyer l'ascenseur social, ils font la dure expérience de l'ambiguïté, pour ne pas dire, comme ils le font parfois, de "l'hypocrisie " du système de solidarité communautaire qui les marginalise et les dévalorise autant qu'il les prend en charge.

En raison, non seulement de leur poids démographique et du sort qui leur est fait à travers cet abandon relatif et forcé, ces jeunes procèdent à des irruptions fracassantes dans un espace social (familial et public) dont ils se sentent exclus et qu'ils «visitent » et investissent fréquemment de manière violente. Cette conflictualité avec la société s'étend également au sein de leurs familles dont ils constituent à la fois les protégés et les boucs émissaires parce que tantôt victimes, tantôt responsables de leur sort, produisant chez eux une ambivalence qui les bascule parfois dans la défiance et le rejet de la famille.

Les logiques de violence, de révolte, de rejet de certaines appartenances (familiale entre autres) et le choix plus ou moins conséquent de la marginalité dans certains cas extrêmes sont à la mesure de ces "traumatismes " que la société leur impose. Ils traduisent au soir de la vie insouciante d'enfants et à l'entrée dans la vie adulte un désenchantement face à la crise d'un système solidaire.

L'interrogation sur les migrations en général et celles des jeunes en particulier, au-delà des mobiles avancés par ces derniers pour justifier le départ en exil, nous conduit à un certain nombre de thèses. Manifestement, parler de misère et de famine comme causes déterminantes de la migration nous a paru exagéré quand on connaît tant soit peu les conditions réelles de vie. Grâce à l'apport des premiers migrants, les villages de la vallée du fleuve Sénégal étaient préservés de la faim et connaissaient des habitudes de consommation de plus en plus modernes et urbaines.

D'un point de vue historique, la migration a assuré la prise en charge de certaines obligations pendant la période coloniale puis postcoloniale (paiement de l'impôt), mais aussi des dépenses modernes acquittées en grande partie grâce au numéraire tiré de l'émigration vers le bassin arachidier sénégalais d'abord (migrations saisonnières des navétanes), vers les autres pays du continent ensuite puis, enfin vers la France. Les obligations matrimoniales (la dot), l'attrait croissant des biens de consommation (habits, postes radio, vélos, produits alimentaires, etc.), la construction de maisons en dur ont alimenté et renforcé le mouvement.

Compte tenu des caractéristiques de l'économie locale (agriculture vivrière essentiellement, circulation monétaire faible ou nulle, enclavement géographique), la migration vers d'autres régions pour se procurer le numéraire s'avérait indispensable à la reproduction sociale. Traite et commerce, navétanat, activité salariée en tant que navigateur (laptot) dans la marine marchande, fluviale puis maritime, ouvrier dans les villes africaines ou en France furent autant de formes historiques et progressives de recherche et de constitution de ces revenus monétarisés. 
De toutes ces formes, l'émigration vers la France semble s'être présentée comme la plus optimale car permettant des gains importants et rapides, comparativement à ceux dégagés par les migrations saisonnières antérieurement pratiquées. Elle tendra à devenir exclusive avec les fermetures sporadiques des autres directions d'émigration, notamment vers l'Afrique centrale (Congo, Zaïre). Certes, les logiques d'inhospitalité et l'expression de phénomènes tels que le mouvement des sans-papiers traduisent néanmoins une forme de «saturation » institutionnelle (précarisation juridique et fabrique à grande échelle des irréguliers) relayée jusqu'à un certain point par une saturation communautaire (mise à distance des irréguliers par leur propre communauté) [Timera, 1997].

Cette recherche du numéraire qui s'est donnée comme le but immédiat de la migration constitue également le moyen de réalisation individuelle du jeune migrant dans le système familial et communautaire, une réponse à la contradiction aînés/cadets. Ainsi, cette dimension individuelle n'est sans doute pas une donnée neuve [Pollet, Winter, 1971], et ces processus d'individuation sont anciens, déjà inscrits dans les sociétés africaines et ont présidé jusqu'à un certain point à l'émigration, ont accompagné le procès d'immigration.

C'est peut-être leur prise en compte qui faisait défaut dans nos outils de réflexion et dans nos manières de construire les objets dans le champ de recherche émigration/immigration. La crise actuelle du système solidaire a sans doute permis de mieux percevoir ce fait et de poser le débat sur les conditions et les moyens d'approcher plus intimement et d'objectiver cette dimension. Le recours à la théorie des réseaux de l'acteur comme nous l'avons tenté dans nos travaux antérieurs n'ayant pas abouti, l'approche biographique ne garantit pas non plus immédiatement une mise en exergue, une objectivation de cette dimension et repose avec acuité la nécessaire reconstruction de l'objet de recherche en fonction de cet objectif de connaissance.

\section{BIBLIOGRAPHIE}

AMSELLE J.-L. [1976], « Aspects et significations du phénomène migratoire en Afrique », in J.-L. Amselle (éd.), Les Migrations africaines. Réseaux et processus migratoires, Paris, Maspero, Dossiers africains: 9-39.

BerTheleu H. [1997] "À propos de l'étude des relations interethniques et du racisme en France », REMI, 13: 117-139.

Daum C., Timera M. [1995], Du village à la ville. Immigration et Développement, Paris, institut Panos.

Frank A.-G. [1970], Le Développement du sous-développement: l'Amérique latine, Paris, Maspero.

Grafmeyer Y., Joseph I. [1990], L'École de Chicago. Naissance de l'écologie urbaine, Paris, AubierChamp urbain, 377 p. (1 $1^{\text {re }}$ éd. 1979).

Marie A. [1997], L'Afrique des individus, Paris, Karthala.

MARIE A. [1998], "Echange: sous le don, la dette ", Sciences humaines, hors série, 23 : 28-31.

Manchuelle F. [1987], Background to Black African Emigration to Francelthe Labor Migrations of the Soninke, 1848-1987, University of California, Ph.D.

Meillassoux C. [1975], Femmes, Greniers et Capitaux, Paris, Maspero (rééd. 1991), L'Harmattan, $251 \mathrm{p}$.

Pollet E., Winter G. [1971], La Société soninké (Dyahunu, Mali), éditions de l'Université libre de Bruxelles, 566 p. 
Quiminal C. [1991], Gens d'ici, Gens d'ailleurs, Paris, Christian Bourgois, 222 p.

RudDER V. DE [1992] «À propos de la construction d'un objet de connaissance: migration et relations interethniques ", Migrants-formation, 90, septembre 1992.

Sayad A. [1991], L'Immigration ou les Paradoxes de l'altérité, Bruxelles, De Boeck-Wesmaël.

SAYad A. [1999], La Double Absence, Paris, Le Seuil.

TALL S.M. [1994], "Les investissements immobiliers à Dakar des émigrants sénégalais ", Revue européenne des migrations internationales, 10 (3): 137-149.

Timera M. [1996], Les Soninké en France. D'une histoire à l'autre, Paris, Karthala, 244 p.

Timera M. [1997], «Sans-papiers africains face aux communautés d'origine », in D. Fassin, A. Morice, C. Quiminal (éd.), Les Lois de l'inhospitalité, Paris, La Découverte: 95-106.

Wieviorka M., [1992], La France raciste, Paris, Le Seuil. 